\title{
Impact of water fortification with calcium on calcium intake in different countries: a simulation study
}

\author{
Gabriela Cormick ${ }^{1,2, *}$, Luz Gibbons ${ }^{1}$ and Jose M Belizán ${ }^{1}$ \\ 'Department of Mother and Child Health Research, Institute for Clinical Effectiveness and Health Policy \\ (IECS-CONICET), Buenos Aires 1414, Argentina: ${ }^{2}$ Departamento de Salud, Universidad Nacional de La Matanza, \\ San Justo 1754, Argentina
}

Submitted 19 September 2019: Final revision received 28 May 2020: Accepted 12 June 2020: First published online 3 August 2020

\begin{abstract}
Objective: To simulate the impact - effectiveness and safety - of water fortification with different concentrations of $\mathrm{Ca}$ using the Intake Modelling, Assessment and Planning Program.

Design: This is a secondary analysis of national or sub-national dietary intake databases.

Setting and Participants: Uganda, Lao People's Democratic Republic (PDR), Bangladesh, Zambia, Argentina, USA and Italy.

Results: We found that for dietary databases assessed from low- and middleincome countries (LMIC), the strategy of fortifying water with $500 \mathrm{mg}$ of $\mathrm{Ca} / \mathrm{l}$ would decrease the prevalence of low Ca intake in all age groups. We also found that this strategy would be safe as no group would present a percentage of individuals exceeding the upper limit in $>2 \%$, except women aged 19-31 years in Lao PDR, where $6.6 \%$ of women in this group would exceed the upper limit of Ca intake. The same strategy would lead to some groups exceeding the upper limit in USA and Italy.

Conclusions: We found that for most LMIC countries, water fortified with Ca could decrease the prevalence of $\mathrm{Ca}$ intake inadequacy without exceeding the upper levels of $\mathrm{Ca}$ intake.
\end{abstract}

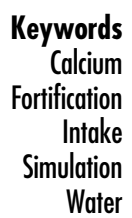

Adequate $\mathrm{Ca}$ intake is associated with bone health, and increasing evidence shows the link between adequate $\mathrm{Ca}$ intake and lower blood pressure particularly among young people, the prevention of hypertensive disorders of pregnancy and lower blood pressure in the progeny of mothers taking sufficient $\mathrm{Ca}$ during pregnancy ${ }^{(1-5)}$. Mechanisms linking low $\mathrm{Ca}$ intake and blood pressure are mediated by parathyroid hormone raise that increases intracellular $\mathrm{Ca}$ in vascular smooth muscle cells leading to vasoconstriction $^{(6)}$. An adequate $\mathrm{Ca}$ intake has also shown to lower cholesterol values and to prevent renal stones and colorectal adenomas ${ }^{(7-10)}$.

Ca intake is low in many countries in the world, in particular, in low-income countries, although low Ca intake can also be found within some population age groups in certain high-income countries (HIC) ${ }^{(5,11-15)}$. The promotion of diets with Ca-rich foods is the ultimate goal. However, a changing dietary habit is difficult to achieve in the short term. In countries where inadequate $\mathrm{Ca}$ intake is widespread, Ca supplements might not be the best option, as they require good adherence, which is often difficult to be achieved by the whole population ${ }^{(16)}$. Furthermore, it has been shown that supplementation adherence is usually better in individuals who already have better micronutrient intake, leading to an improvement of the population diet but not necessarily in those more at need ${ }^{(17)}$. Therefore, food fortification strategies seem to be a good option, although they require appropriate planning to determine the food fortification level necessary to improve Ca intake without putting any population group at risk of excess ${ }^{(18)}$. Fortification planning requires data on food and nutrient intake representative of the aimed population so as to define the level of nutrient inadequacy in the population and then decide the appropriate food vehicle that can be fortified $^{(18)}$. The next step is a simulation of the impact of different fortification levels on the distribution of nutrient 
intake of each population group that will be reached by the fortification strategy. This step allows to estimate the reduction of nutrient inadequacy for each population group as well as the percentage of individuals with excess intake.

Traditionally, fortification vehicles have been staple foods and condiments such as salt as these foods are vastly consumed ensuring to reach all individuals in a given population. Although less explored as a fortification vehicle, water has been used in fluoride fortification ${ }^{(19)}$. Ca fortification of water seems a promising approach as water is universally consumed and $\mathrm{Ca}$ in water has a good bioavailability ${ }^{(20-22)}$. Ca bioavailability from Ca-rich waters is similar to that of milk ${ }^{(20-22)}$. Ca absorption might be increased in certain groups such as pregnant women, for that reason some countries do not increase the recommendation of $\mathrm{Ca}$ intake ${ }^{(23)}$. Furthermore, Ca bioavailability is improved when $\mathrm{Ca}$ intake is spread in low doses throughout the day rather than in one load. In this way, water as a vehicle seems a good alternative even if the Ca content in water is not very high ${ }^{(24)}$. Another benefit of using water as a fortification vehicle is that increasing water intake is also a beneficial public health promotion message, whereas other vehicles such as salt or wheat flour might have some consumption restrictions for certain population groups with hypertension or food allergies. Even more, increasing water intake is part of a healthy diet and taking into account the global obesity epidemic increasing water intake does not comprise a change in the total energy intake.

The IOWA University has developed the Intake Modelling Assessment Program (IMAPP), a computer programme that allows running the simulation of different fortification scenarios using the information of population daily nutrient intake. The programme allows estimating the best amount of a fortificant to be added to a food vehicle in order to decrease the level of nutrient inadequacy, without exceeding the recommended upper limits ${ }^{(25,26)}$.

The objective of the current study is to simulate the impact - effectiveness and safety - of water fortification with Ca using the available dietary intake information from Uganda, Lao People's Democratic Republic (PDR), Bangladesh, Zambia, Argentina, Italy and USA ${ }^{(11)}$. These countries show a diversity of situations involving lowand middle-income countries (LMIC), one from South America, two from Africa, two from Asia and two HIC, one from North America and one from Europe.

\section{Materials and methods}

We search for available national or sub-national dietary assessment databases collected through 24-h recalls or dietary records, we analysed all publicly available. This is an analysis of dietary intake databases of 24-h recalls from Uganda, Lao PDR, Bangladesh, Zambia, Argentina and USA and of self-recorded food records from Italy. Uganda, Lao PDR, Bangladesh, Zambia and Argentina are countries in regions with very low Ca intake (below $400 \mathrm{mg} / \mathrm{d}$ ), whereas the USA and Italy show an overall adequate Ca intake $^{(11)}$. Databases from Uganda, Lao PDR, Bangladesh, Zambia and Italy were obtained from the FAO/WHO Global Individual Food consumption data Tool, an openaccess online platform, hosted by FAO and supported by $\mathrm{WHO}^{(27)}$. Argentina's database was obtained from the Ministry of Health, and the USA's database was obtained from the Centers for Diseases Control and Prevention website $^{(28,29)}$.

For each database, we evaluated the effectiveness and safety of different Ca fortification scenarios with the aim of shifting the distribution of Ca intake so that the majority of population groups improve their $\mathrm{Ca}$ intake and achieve a mean intake closer to their requirement without exceeding the recommended upper limit ${ }^{(25)}$. Effectiveness was measured as the percentage of individuals below the Institute of Medicine (IOM) estimated average requirement (EAR) and safety as the percentage of individuals exceeding the IOM upper limit (UL) of their corresponding age-specific population subgroup.

The Ca EAR defined by the IOM is $270 \mathrm{mg} / \mathrm{d}$ for infants $<1$ year, $400 \mathrm{mg} / \mathrm{d}$ for children aged 1 to $<4$ years, $640 \mathrm{mg} / \mathrm{d}$ for children aged 4 to $<9$ years and $1100 \mathrm{mg} / \mathrm{d}$ for those aged 9 to $<19$ years. The EAR for Ca defined by the IOM is $800 \mathrm{mg} / \mathrm{d}$ for those aged 19 to $<51$ years, $800 \mathrm{mg} / \mathrm{d}$ for males aged 51 to $<71$ years and $1000 \mathrm{mg} / \mathrm{d}$ for women aged $\geq 51$ years and men $\geq 71$ years. The Ca UL defined by the IOM is $1500 \mathrm{mg} / \mathrm{d}$ for infants < 1 year, $2500 \mathrm{mg} / \mathrm{d}$ for children aged 1 to $<9$ years, $3000 \mathrm{mg} / \mathrm{d}$ for those aged 9 to $<19$ years, $2500 \mathrm{mg} / \mathrm{d}$ for those aged 19 to $<51$ years and $2000 \mathrm{mg} / \mathrm{d}$ for those aged $\geq 51$ years. Pregnant and lactating women aged 14-50 years have the same EAR and UL as those of nonpregnant women.

\section{Population}

\section{Uganda}

We used dietary intake data from the baseline survey of the HarvestPlus Reaching End Users Orange-Fleshed Sweet Potato project performed in three rural regions of Eastern and Central Uganda: Bukedea, Kamuli and Mukono. This cross-sectional study too place from 1st January to 31st December 2007. The project aimed at inducing broad Orange-Fleshed Sweet Potato adoption to increase vitamin A intakes and reduce vitamin A deficiency among women in Uganda ${ }^{(27)}$. The survey included 577 women of reproductive age. After classifying participants into the IOM age categories for dietary recommended values, we found that only three girls were younger than 19 years, so they were excluded from the analysis. Nine of those had missing data, and they were also excluded. A total of 565 women were included in the dietary intake analysis, 270 nonpregnant women aged 20-67 years and 295 pregnant women aged $19-48$ years. 


\section{Lao People's Democratic Republic}

We used data from the National Food Consumption Survey Lao People's Democratic Republic performed in rural and urban settings from December 2016 to May 2017 to cover both the dry and rainy seasons ${ }^{(27)}$. The survey included 2045 individuals, 870 males and 1175 women. According to the IOM age categories, we found a few number of individuals in the groups of pregnant women aged 51 or above, non-pregnant women aged 71 or above and boys aged 9 to $<19$ years so they were excluded from this analysis. A total of 1919 individuals were analysed in the current study, 1048 children aged 3 months to < 14 years, 319 nonpregnant girls and women aged 14-86 years, 285 pregnant women aged $16-56$ years and 267 men aged $19-89$ years.

\section{Bangladesh}

We used data from the initial survey of a HarvestPlus multistage research programme to determine the potential impact of $\mathrm{Zn}$-biofortified rice on the $\mathrm{Zn}$ and health status among children in Bangladesh. The original study was conducted in collaboration with the University of California, Davis and the International Centre for Diarrhoel Disease Research, Bangladesh (ICDDR) from 30th September 2007 to 29th June 2008. Dietary information was collected primarily by direct observation and weighing of food preparation and consumption ${ }^{(27)}$.

According to the IOM age categories, there were 224 non-pregnant women aged $18-70$ years and 236 pregnant women aged 5-50 years. The survey also included girls aged 14-19 years and women aged 51 to $<71$ years; however, as there were very few cases in those groups, we were not able to analyse them.

\section{Zambia}

We used data from the initial survey of the HarvestPlus nutritional survey. This survey was carried out in Nyimba District in Eastern Province and Mkushi District in Central Province with the collaboration with the National Food and Nutrition Commission (Lusaka, Zambia) and the Tropical Diseases Research Centre from 30 April 2007 to 29 December 2009. The main goal of the survey was to obtain adequate background nutritional information among preschool children from rural Zambia to assess the potential impact of food-based interventions to improve vitamin A status, including provitamin A-biofortified maize ${ }^{(27)}$.

According to the IOM age categories, there were 454 children aged 1 to <9 years, 145 non-pregnant women aged 19 to $<50$ years and 186 pregnant women aged 17 to $<51$ years. The survey also included infants $<1$ year and girls aged 14 to $<19$ years (pregnant or not) and women (pregnant or not) aged 51 to $<71$ years; however, there were very few cases in those groups and we were not able to analyse them.

\section{Argentina}

We used data from the first Health and Nutrition National Survey carried out by the Ministry of Health in Argentina between 2004 and 2005(28). Participants were selected using a probabilistic complex sample design including different socioeconomic levels from both large and small cities of all provinces of Argentina representing the whole population $^{(30)}$. Data were collected using a single $24 \mathrm{~h}$ recall.

The survey included 1338 children aged 0 to $<9$ years, 6605 women aged 19 to $<50$ years and 1610 pregnant women 14 to $<45$ years.

\section{Italy}

We used data from the third national survey L'indagine nazionale sui consumi alimentari in Italia performed by the Italian Consiglio per la ricerca in agricoltura e l'analisi dell'economia agrarian from October 2005 to December $2006^{(31)}$. The survey covered all seasons and included a sample representative of the North-West, North East, Centre, South and Islands of Italy. Consumption of all foods, beverages, food supplements and medicines was self-recorded by subjects for three consecutive days on hard-copy diaries structured by meal. For our analysis, we used the first and third survey days.

The survey included 3323 individuals 1501 men and 1793 women; however, some had not all required data, and finally, 3269 were analysed. According to the IOM age categories, there were 377 children aged 0 to $<14$ years, 28 pregnant women aged 19 to <51 years, 1584 non-pregnant women aged 19 to <97 years and 1280 men aged 19 to <92 years.

USA

We used data from the 2016 nationally representative, cross-sectional survey of the non-institutionalised US population National Health and Nutrition Examination Survey (NHANES) administered by the National Center for Health Statistics within the $\mathrm{CDC}^{(29)}$. The survey has a stratified, multistage probability cluster sampling design.

The survey included 9971 individuals, however, 8339 had data on Ca intake, 2443 children aged 0 to $<19$ years, 2974 women aged 19 to <80 years, 2859 men aged 19 to $<80$ years and sixty-three pregnant women 20 to $<42$ years.

\section{Analysis}

Ca intake was estimated using the daily intake information from individuals in each of the selected countries. Each individual was classified according to the IOM dietary reference values age-specific groups: children, adolescents, adults and pregnant women. Afterwards, we determined how many individuals were in each population group and how many had repeated dietary intake information. 
We estimated day-to-day Ca intake variability of for those groups that had a repeated dietary intake data in non-consecutive days. The variability was then used to adjust the Ca intake distribution of one single day in order to obtain an estimated distribution of Ca usual intake. If repeated dietary intake data were not available, we estimated the usual $\mathrm{Ca}$ intake using a default external variance ratio for Ca provided by the IMAPP.

We used IMAPP to calculate the baseline prevalence of inadequate $\mathrm{Ca}$ intake using the corresponding EAR and UL for $\mathrm{Ca}$ defined by IOM for each population group. Prevalence of low Ca intake was calculated as the proportion of individuals in the group with usual $\mathrm{Ca}$ intake below the age-specific EAR, and prevalence of excess was calculated as the proportion of individuals with usual Ca intakes above the age-specific $\mathrm{UL}^{(32)}$.

After calculating the prevalence of $\mathrm{Ca}$ intake inadequacy for each population group, we selected the group with the highest prevalence of low $\mathrm{Ca}$ intake and defined a desired 'target prevalence of inadequate intake' to plan the water fortification simulation.

We then calculated the initial gap defined as the estimated amount of Ca that should be added to water in order to achieve the desired 'target prevalence of inadequate intakes'. The initial gap was calculated as difference in $\mathrm{mg} / \mathrm{d}$ between the desired prevalence of that group.

Subsequently, using IMAPP, we estimated the Ca intake distributions after simulating the addition of $\mathrm{Ca}$ to each individual water intake. In the case that the dietary information did not contain the amount of water intake, we simulated two scenarios: the consumption of 1 and then 1.5 litres of water intake per day for individuals older than 1 year and 0.6 and 0.8 litres of water intake per day for infants aged 6 to $<12$ months. We first simulated adding $500 \mathrm{mg}$ of elemental $\mathrm{Ca} / \mathrm{l}$ of water. We selected $500 \mathrm{mg}$ of $\mathrm{Ca}$ as it is the approximate gap between $\mathrm{Ca}$ intake in LMIC and $\mathrm{HIC}^{(5,12)}$. In cases where the percentage of individuals exceeded the upper limit above $2.5 \%$ in any of the groups, we decreased the amount of $\mathrm{Ca}$ to 400,300 or $200 \mathrm{mg} / \mathrm{l}$ of water. We then re-calculated the prevalence of $\mathrm{Ca}$ inadequacy of each population group in the database after simulating the addition of different amounts of Ca to water.

\section{Results}

The number of participants for each age group, prevalence of low $\mathrm{Ca}$ intake and percentage of individuals exceeding the upper limit as well as the changes on $\mathrm{Ca}$ intake after simulating the addition of $\mathrm{Ca}$ to water for each country are described in Tables 1-7. The only databases with information on water intake were those from the USA and Italy, and for the rest of the countries, Uganda, Lao PDR, Bangladesh, Zambia, Italy and Argentina, we simulated an intake of 1 and 1.5 litres of water/individual per $\mathrm{d}$. 
Table 2 Simulation of the effect of water fortification with $500 \mathrm{mg}$ of calcium/l on calcium intake (Lao PDR)

\begin{tabular}{|c|c|c|c|c|c|c|c|c|c|c|c|c|c|c|}
\hline \multirow[b]{2}{*}{ Group } & \multirow[b]{2}{*}{$n$} & \multirow[b]{2}{*}{$\operatorname{EAR}(\mathrm{mg})$} & \multicolumn{4}{|c|}{ Basal Ca intake } & \multicolumn{4}{|c|}{$\begin{array}{c}\text { Ca intake after simulation of intake of } \\
1 \text { litre of fortified water a day }\end{array}$} & \multicolumn{4}{|c|}{$\begin{array}{c}\text { Ca intake after simulation of intake of } \\
1.5 \text { litre of fortified water a day }\end{array}$} \\
\hline & & & Mean (mg) & SD & $<\operatorname{EAR}(\%)$ & $>\mathrm{UL}(\%)$ & Mean (mg) & SD & $<\operatorname{EAR}(\%)$ & $>$ UL (\%) & Mean $(\mathrm{mg})$ & SD & $<\operatorname{EAR}(\%)$ & $>$ UL (\%) \\
\hline \multicolumn{15}{|l|}{ Children } \\
\hline $0.5 \leq$ age $<1$ & 170 & 270 & 328.8 & 398.6 & $62 \cdot 3$ & $2 \cdot 0$ & $629 \cdot 0^{*}$ & 371.9 & 0.0 & 3.2 & $728 \cdot 7 \dagger$ & 363.7 & 0.0 & 3.8 \\
\hline $1 \leq \overline{a g e}<4$ & 407 & 400 & 364.1 & 257.9 & $66 \cdot 8$ & 0.0 & $866 \cdot 9$ & $267 \cdot 9$ & 0.0 & 0.0 & 1116.7 & $267 \cdot 3$ & 0.0 & 0.2 \\
\hline $4 \leq$ age $<9$ & 294 & 640 & $214 \cdot 1$ & 104.6 & 99.5 & 0.0 & $713 \cdot 0$ & $100 \cdot 4$ & $24 \cdot 8$ & 0.0 & $962 \cdot 4$ & $99 \cdot 6$ & 0.0 & 0.0 \\
\hline \multicolumn{15}{|c|}{ Women non-pregnant } \\
\hline $9 \leq$ age $<14$ & 74 & 1100 & $207 \cdot 2$ & 93.4 & $100 \cdot 0$ & 0.0 & 704.6 & 83.9 & 99.9 & 0.0 & 954.9 & 83.8 & 93.5 & 0.0 \\
\hline $19 \leq$ age $<31$ & 53 & 800 & 263.5 & 109.9 & $99 \cdot 8$ & 0.0 & 761.5 & $108 \cdot 6$ & $71 \cdot 6$ & 0.0 & $1010 \cdot 7$ & $107 \cdot 6$ & 0.0 & 0.0 \\
\hline $31 \leq$ age $<51$ & 101 & 800 & $250 \cdot 6$ & 105.4 & $99 \cdot 8$ & 0.0 & 749.9 & $102 \cdot 3$ & $75 \cdot 7$ & 0.0 & 999.9 & 101.9 & 0.0 & 0.0 \\
\hline $51 \leq$ age $<71$ & 127 & 1000 & $230 \cdot 0$ & $125 \cdot 3$ & 99.9 & 0.0 & $736 \cdot 8$ & 138.9 & 94.9 & 0.0 & $990 \cdot 3$ & $148 \cdot 2$ & 63.4 & 0.1 \\
\hline \multicolumn{15}{|l|}{ Pregnant women } \\
\hline $14 \leq$ age $<19$ & 23 & 1100 & $229 \cdot 6$ & $103 \cdot 1$ & $100 \cdot 0$ & 0.0 & $754 \cdot 6$ & 91.7 & $99 \cdot 8$ & 0.0 & $1004 \cdot 7$ & 91.6 & $85 \cdot 3$ & 0.0 \\
\hline $19 \leq$ age $<31$ & 196 & 800 & $313 \cdot 7$ & $185 \cdot 5$ & 97.6 & 0.0 & 820.7 & 189.7 & $55 \cdot 7$ & 0.0 & $1067 \cdot 7$ & $186 \cdot 3$ & 0.2 & 0.0 \\
\hline $31 \leq$ age $<51$ & 66 & 800 & $300 \cdot 6$ & $167 \cdot 1$ & $98 \cdot 3$ & 0.0 & $798 \cdot 1$ & $151 \cdot 8$ & $60 \cdot 0$ & 0.0 & $1046 \cdot 8$ & $149 \cdot 3$ & 0.0 & 0.0 \\
\hline \multicolumn{15}{|l|}{ Male } \\
\hline $19 \leq$ age $<31$ & 22 & 800 & $212 \cdot 0$ & 62.5 & $100 \cdot 0$ & 0.0 & $712 \cdot 2$ & $63 \cdot 2$ & $90 \cdot 8$ & $0 \cdot 0$ & $962 \cdot 3$ & 63.6 & $0 \cdot 0$ & $0 \cdot 0$ \\
\hline $31 \leq$ age $<51$ & 108 & 800 & $435 \cdot 6$ & 381.3 & 89.7 & 0.5 & 903.2 & 283.0 & $45 \cdot 0$ & 0.3 & $1149 \cdot 3$ & 274.1 & 0.0 & 0.5 \\
\hline
\end{tabular}

EAR, estimated average requirement; UL, upper limit.

*Assuming $600 \mathrm{ml}$ of water intake/d. 
Table 3 Simulation of the effect of water fortification with $500 \mathrm{mg}$ of calcium/l on calcium intake (Bangladesh)

\begin{tabular}{|c|c|c|c|c|c|c|c|c|c|c|c|c|c|c|}
\hline \multirow[b]{2}{*}{ Group } & \multirow[b]{2}{*}{$n$} & \multirow[b]{2}{*}{$\begin{array}{l}\text { EAR } \\
(\mathrm{mg})\end{array}$} & \multicolumn{4}{|c|}{ Basal Ca intake } & \multicolumn{4}{|c|}{$\begin{array}{c}\text { Ca intake after simulation of } \\
\text { intake of } 1 \text { litre of fortified water } \\
\text { a day }\end{array}$} & \multicolumn{4}{|c|}{$\begin{array}{c}\text { Ca intake after simulation of } \\
\text { intake of } 1.5 \text { litre of fortified } \\
\text { water a day }\end{array}$} \\
\hline & & & $\begin{array}{c}\text { Mean } \\
(\mathrm{mg})\end{array}$ & SD & $\begin{array}{c}<\text { EAR } \\
(\%)\end{array}$ & $\begin{array}{l}>U L \\
(\%)\end{array}$ & $\begin{array}{c}\text { Mean } \\
(\mathrm{mg})\end{array}$ & SD & $\begin{array}{c}<\text { EAR } \\
(\%)\end{array}$ & $\begin{array}{l}>U L \\
(\%)\end{array}$ & $\begin{array}{c}\text { Mean } \\
(\mathrm{mg})\end{array}$ & SD & $\begin{array}{c}<\text { EAR } \\
(\%)\end{array}$ & $\begin{array}{c}>U L \\
(\%)\end{array}$ \\
\hline \multicolumn{15}{|c|}{ Women non-pregnant } \\
\hline $19 \leq$ age $<31$ & 157 & 800 & $160 \cdot 8$ & $55 \cdot 6$ & $100 \cdot 0$ & 0.0 & 660.5 & $56 \cdot 1$ & $97 \cdot 6$ & 0.0 & 910.9 & 55.9 & 0.0 & 0.0 \\
\hline $31 \leq$ age $<51$ & 67 & 800 & $151 \cdot 8$ & 53.7 & $100 \cdot 0$ & 0.0 & $650 \cdot 8$ & $55 \cdot 0$ & $98 \cdot 3$ & 0.0 & 901.4 & $55 \cdot 3$ & 0.2 & 0.0 \\
\hline \multicolumn{15}{|l|}{ Pregnant women } \\
\hline $19 \leq$ age $<31$ & 174 & 800 & $150 \cdot 2$ & $58 \cdot 8$ & $100 \cdot 0$ & 0.0 & $653 \cdot 1$ & $70 \cdot 8$ & $95 \cdot 8$ & 0.0 & 903.5 & $71 \cdot 2$ & 0.0 & 0.0 \\
\hline $31 \leq$ age $<51$ & 62 & 800 & $143 \cdot 2$ & $80 \cdot 3$ & $100 \cdot 0$ & $0 \cdot 0$ & $635 \cdot 5$ & $69 \cdot 2$ & $96 \cdot 9$ & 0.0 & 885.9 & $69 \cdot 2$ & 0.8 & 0.0 \\
\hline
\end{tabular}

EAR, estimated average requirement; UL, upper limit.

Table 4 Simulation of the effect of water fortification with $500 \mathrm{mg}$ of calcium/l on calcium intake (Zambia)

\begin{tabular}{|c|c|c|c|c|c|c|c|c|c|c|c|c|c|c|}
\hline \multirow[b]{2}{*}{ Group } & \multirow[b]{2}{*}{$n$} & \multirow[b]{2}{*}{$\begin{array}{l}\text { EAR } \\
(\mathrm{mg})\end{array}$} & \multicolumn{4}{|c|}{ Basal Ca intake } & \multicolumn{4}{|c|}{$\begin{array}{c}\text { Ca intake after simulation of } \\
\text { intake of } 1 \text { litre of fortified water } \\
\text { a day }\end{array}$} & \multicolumn{4}{|c|}{$\begin{array}{c}\text { Ca intake after simulation of } \\
\text { intake of } 1.5 \text { litre of fortified water } \\
\text { a day }\end{array}$} \\
\hline & & & $\begin{array}{c}\text { Mean } \\
(\mathrm{mg})\end{array}$ & SD & $\begin{array}{c}<\text { EAR } \\
(\%)\end{array}$ & $\begin{array}{c}>U L \\
(\%)\end{array}$ & $\begin{array}{c}\text { Mean } \\
(\mathrm{mg})\end{array}$ & SD & $\begin{array}{c}<\text { EAR } \\
(\%)\end{array}$ & $\begin{array}{c}>U L \\
(\%)\end{array}$ & $\begin{array}{c}\text { Mean } \\
(\mathrm{mg})\end{array}$ & SD & $\begin{array}{c}<\text { EAR } \\
(\%)\end{array}$ & $\begin{array}{c}>U L \\
(\%)\end{array}$ \\
\hline \multicolumn{15}{|l|}{ Children } \\
\hline $1 \leq$ age $<4$ & 322 & 400 & $202 \cdot 6$ & $70 \cdot 8$ & 98.7 & 0.0 & $702 \cdot 6$ & 71.0 & 0.0 & 0.0 & $952 \cdot 7$ & $70 \cdot 8$ & 0.0 & 0.0 \\
\hline $4 \leq$ age $<9$ & 132 & 640 & $220 \cdot 2$ & $64 \cdot 0$ & $100 \cdot 0$ & 0.0 & 720.6 & $62 \cdot 0$ & $6 \cdot 9$ & 0.0 & 970.9 & 61.8 & 0.0 & 0.0 \\
\hline \multicolumn{15}{|c|}{ Women non-pregnant } \\
\hline $19 \leq$ age $<31$ & 73 & 800 & 339.3 & $9 \cdot 6$ & $100 \cdot 0$ & 0.0 & $840 \cdot 0$ & $6 \cdot 1$ & 0.0 & 0.0 & $1089 \cdot 3$ & 7.8 & 0.0 & 0.0 \\
\hline $31 \leq$ age $<51$ & 72 & 800 & 311.5 & 24.5 & $100 \cdot 0$ & 0.0 & 811.5 & 33.4 & 38.3 & 0.0 & 1061.8 & $35 \cdot 6$ & 0.0 & 0.0 \\
\hline \multicolumn{15}{|l|}{ Pregnant women } \\
\hline $19 \leq$ age $<31$ & 126 & 800 & $322 \cdot 2$ & 114.4 & $99 \cdot 8$ & 0.0 & $821 \cdot 0$ & $109 \cdot 6$ & $47 \cdot 6$ & 0.0 & 1071.0 & $108 \cdot 8$ & 0.0 & 0.0 \\
\hline $31 \leq$ age $<51$ & 60 & 800 & 317.4 & 85.6 & $100 \cdot 0$ & 0.0 & $817 \cdot 0$ & $88 \cdot 8$ & 45.4 & 0.0 & $1067 \cdot 1$ & 89.2 & 0.0 & 0.0 \\
\hline
\end{tabular}

EAR, estimated average requirement; UL, upper limit.

Table 5 Simulation of the effect of water fortification with $500 \mathrm{mg}$ of calcium/l on calcium intake (Argentina)

\begin{tabular}{|c|c|c|c|c|c|c|c|c|c|c|c|c|c|c|}
\hline \multirow[b]{2}{*}{ Group } & \multirow[b]{2}{*}{$n$} & \multirow[b]{2}{*}{$\begin{array}{l}\text { EAR } \\
(\mathrm{mg})\end{array}$} & \multicolumn{4}{|c|}{ Basal Ca intake } & \multicolumn{4}{|c|}{$\begin{array}{c}\text { Ca intake after simulation of } \\
\text { intake of } 1 \text { litre of fortified water } \\
\text { a day }\end{array}$} & \multicolumn{4}{|c|}{$\begin{array}{c}\text { Ca intake after simulation of } \\
\text { intake of } 1.5 \text { litre of fortified } \\
\text { water a day }\end{array}$} \\
\hline & & & $\begin{array}{c}\text { Mean } \\
(\mathrm{mg})\end{array}$ & SD & $\begin{array}{c}<\mathrm{EAR} \\
(\%)\end{array}$ & $\begin{array}{l}>U L \\
(\%)\end{array}$ & $\begin{array}{l}\text { Mean } \\
(\mathrm{mg})\end{array}$ & SD & $\begin{array}{c}<\mathrm{EAR} \\
(\%)\end{array}$ & $\begin{array}{l}>U L \\
(\%)\end{array}$ & $\begin{array}{l}\text { Mean } \\
(\mathrm{mg})\end{array}$ & SD & $\begin{array}{c}<\mathrm{EAR} \\
(\%)\end{array}$ & $\begin{array}{l}>U L \\
(\%)\end{array}$ \\
\hline \multicolumn{15}{|l|}{ Children } \\
\hline $0.5 \leq$ age $<1$ & 2110 & 270 & $609 \cdot 3$ & $348 \cdot 0$ & $17 \cdot 5$ & 1.5 & $909 \cdot 6^{*}$ & $348 \cdot 2$ & 0.0 & $6 \cdot 1$ & $1009 \cdot 6 \dagger$ & $348 \cdot 1$ & 0.0 & $9 \cdot 3$ \\
\hline $1 \leq$ age $<4$ & 7787 & 400 & $752 \cdot 2$ & 323.4 & $13 \cdot 4$ & 0.0 & 1252.5 & $324 \cdot 3$ & 0.0 & $0 \cdot 1$ & $1502 \cdot 5$ & $324 \cdot 3$ & 0.0 & 0.5 \\
\hline $4 \leq$ age $<9$ & 3480 & 640 & 683.3 & $260 \cdot 6$ & $46 \cdot 2$ & 0.0 & $1182 \cdot 5$ & 258.3 & 0.4 & 0.0 & $1432 \cdot 5$ & 258.3 & 0.0 & 0.0 \\
\hline \multicolumn{15}{|c|}{ Women non-pregnant } \\
\hline $9 \leq$ age $<14$ & 864 & 1100 & $460 \cdot 7$ & $167 \cdot 6$ & 99.9 & 0.0 & $938 \cdot 1$ & $197 \cdot 8$ & $80 \cdot 6$ & 0.0 & $1188 \cdot 1$ & $197 \cdot 7$ & $27 \cdot 6$ & 0.0 \\
\hline $14 \leq$ age $<19$ & 1122 & 1100 & $438 \cdot 8$ & $198 \cdot 2$ & $99 \cdot 4$ & 0.0 & $902 \cdot 0$ & $168 \cdot 1$ & $81 \cdot 0$ & 0.0 & $1152 \cdot 0$ & 167.9 & $37 \cdot 7$ & 0.0 \\
\hline $19 \leq$ age $<31$ & 2112 & 800 & $402 \cdot 6$ & $168 \cdot 1$ & $97 \cdot 6$ & 0.0 & $862 \cdot 1$ & $180 \cdot 0$ & 30.4 & 0.0 & $1112 \cdot 4$ & $180 \cdot 1$ & 0.0 & 0.0 \\
\hline $31 \leq$ age $<51$ & 2507 & 800 & 361.6 & $180 \cdot 2$ & $97 \cdot 6$ & 0.0 & 960.4 & $166 \cdot 8$ & $42 \cdot 7$ & 0.0 & 1210.5 & $166 \cdot 7$ & 0.0 & 0.0 \\
\hline \multicolumn{15}{|l|}{ Pregnant women } \\
\hline $14 \leq$ age $<19$ & 197 & 1100 & $470 \cdot 6$ & 246.7 & $97 \cdot 8$ & 0.0 & $970 \cdot 8$ & 248.0 & $75 \cdot 6$ & 0.0 & $1219 \cdot 8$ & 244.9 & 35.9 & 0.0 \\
\hline $19 \leq$ age $<31$ & 963 & 800 & $490 \cdot 5$ & 240.1 & $89 \cdot 0$ & 0.0 & 990.5 & $240 \cdot 0$ & $23 \cdot 1$ & 0.0 & 1240.5 & 239.9 & 0.0 & 0.0 \\
\hline $31 \leq$ age $<51$ & 450 & 800 & 491.3 & 244.7 & 88.4 & 0.0 & 991.3 & $245 \cdot 3$ & $24 \cdot 2$ & 0.0 & 1241.4 & 245.4 & 0.0 & 0.0 \\
\hline
\end{tabular}

EAR, estimated average requirement; UL, upper limit.

${ }^{*}$ Assuming $600 \mathrm{ml}$ of water intake/d.

†Assuming $800 \mathrm{ml}$ of water intake/d. 
Table 6 Simulation of the effect of water fortification with $500 \mathrm{mg}$ of calcium/l on calcium intake (Italy)

\begin{tabular}{|c|c|c|c|c|c|c|c|c|c|c|c|c|c|c|c|}
\hline \multirow[b]{2}{*}{ Group } & \multirow[b]{2}{*}{$n$} & \multirow[b]{2}{*}{$\begin{array}{l}\text { EAR } \\
(\mathrm{mg})\end{array}$} & \multirow[b]{2}{*}{$\begin{array}{l}\text { Water } \\
\text { Intake }\end{array}$} & \multicolumn{4}{|c|}{ Basal Ca intake } & \multicolumn{4}{|c|}{$\begin{array}{l}\text { Ca intake after fortification of } \\
\text { water with } 500 \mathrm{mg} \text { of } \mathrm{Ca} / \mathrm{l}\end{array}$} & \multicolumn{4}{|c|}{$\begin{array}{l}\text { Ca intake after fortification of } \\
\text { water with } 400 \mathrm{mg} \text { of } \mathrm{Ca} / \mathrm{l}\end{array}$} \\
\hline & & & & $\begin{array}{l}\text { Mean } \\
(\mathrm{mg})\end{array}$ & SD & $\begin{array}{c}<\mathrm{EAR} \\
(\%)\end{array}$ & $\begin{array}{l}>U L \\
(\%)\end{array}$ & $\begin{array}{l}\text { Mean } \\
(\mathrm{mg})\end{array}$ & SD & $\begin{array}{c}<\mathrm{EAR} \\
(\%)\end{array}$ & $\begin{array}{l}>\mathrm{UL} \\
(\%)\end{array}$ & $\begin{array}{l}\text { Mean } \\
(\mathrm{mg})\end{array}$ & SD & $\begin{array}{c}<\mathrm{EAR} \\
(\%)\end{array}$ & $\begin{array}{l}>\mathrm{UL} \\
(\%)\end{array}$ \\
\hline \multicolumn{16}{|l|}{ Children } \\
\hline $0 \leq$ age $<1$ & 13 & 270 & 444.0 & 577.0 & $127 \cdot 5$ & 0.3 & 0.0 & $790 \cdot 6$ & $205 \cdot 8$ & 0.0 & 0.0 & 747.5 & $182 \cdot 4$ & 0.0 & 0.0 \\
\hline $1 \leq$ age $<4$ & 53 & 400 & $415 \cdot 3$ & 728.6 & $180 \cdot 5$ & 1.9 & 0.0 & $930 \cdot 2$ & $221 \cdot 1$ & 0.1 & 0.0 & 889.9 & 211.4 & 0.2 & 0.0 \\
\hline $4 \leq$ age $<9$ & 145 & 640 & $513 \cdot 6$ & 725.9 & $210 \cdot 6$ & $37 \cdot 2$ & 0.0 & 974.6 & $255 \cdot 0$ & $6 \cdot 6$ & 0.0 & 924.8 & 244.2 & $9 \cdot 6$ & 0.0 \\
\hline \multicolumn{16}{|c|}{ Women pregnant } \\
\hline $19 \leq$ age $<31$ & 4 & 800 & $1146 \cdot 9$ & $1009 \cdot 1$ & $181 \cdot 2$ & $13 \cdot 1$ & 0.0 & $1363 \cdot 2$ & $134 \cdot 9$ & 0.0 & 0.0 & $1355 \cdot 8$ & $121 \cdot 1$ & 0.0 & 0.0 \\
\hline $31 \leq$ age $<51$ & 24 & 800 & $823 \cdot 6$ & $822 \cdot 0$ & $267 \cdot 6$ & $51 \cdot 0$ & 0.0 & $1257 \cdot 1$ & $374 \cdot 1$ & $9 \cdot 4$ & 0.4 & $1169 \cdot 9$ & $350 \cdot 3$ & $13 \cdot 6$ & 0.2 \\
\hline \multicolumn{16}{|c|}{ Women non-pregnant } \\
\hline $9 \leq$ age $<14$ & 83 & 1100 & $592 \cdot 1$ & $791 \cdot 2$ & $247 \cdot 8$ & 88.9 & 0.0 & $1090 \cdot 7$ & $316 \cdot 1$ & $57 \cdot 2$ & 0.0 & $1030 \cdot 9$ & 299.4 & 64.7 & 0.0 \\
\hline $14 \leq$ age $<19$ & 84 & 1100 & $662 \cdot 1$ & $819 \cdot 5$ & $187 \cdot 0$ & $92 \cdot 3$ & 0.0 & $1151 \cdot 1$ & $270 \cdot 3$ & $46 \cdot 6$ & 0.0 & 1084.5 & 248.9 & $56 \cdot 7$ & 0.0 \\
\hline $19 \leq$ age $<31$ & 261 & 800 & 789.8 & $716 \cdot 4$ & 237.5 & 68.4 & 0.0 & $1105 \cdot 0$ & 340.2 & $18 \cdot 6$ & 0.1 & $1027 \cdot 1$ & 314.1 & 24.8 & 0.0 \\
\hline $31 \leq$ age $<51$ & 551 & 800 & $683 \cdot 1$ & 734.9 & $236 \cdot 3$ & $65 \cdot 7$ & 0.0 & $1078 \cdot 3$ & 309.5 & $17 \cdot 8$ & 0.0 & $1009 \cdot 6$ & 287.9 & $23 \cdot 8$ & 0.0 \\
\hline $51 \leq$ age $<71$ & 482 & 1000 & $695 \cdot 7$ & $756 \cdot 0$ & 243.9 & $84 \cdot 7$ & 0.0 & 1103.4 & 323.8 & $41 \cdot 0$ & $1 \cdot 0$ & $1033 \cdot 8$ & $303 \cdot 2$ & $49 \cdot 7$ & 0.5 \\
\hline $71 \leq$ age & 206 & 1000 & $661 \cdot 8$ & 799.7 & 228.4 & 81.8 & 0.0 & 1141.9 & $306 \cdot 7$ & 34.6 & 0.8 & 1073.5 & $287 \cdot 7$ & $43 \cdot 1$ & 0.4 \\
\hline \multicolumn{16}{|l|}{ Male } \\
\hline $9 \leq$ age $<14$ & 83 & 1100 & $627 \cdot 5$ & 881.9 & $325 \cdot 0$ & $78 \cdot 0$ & 0.0 & $1189 \cdot 7$ & 383.8 & $45 \cdot 5$ & 0.0 & $1128 \cdot 1$ & 369.7 & $52 \cdot 3$ & 0.0 \\
\hline $14 \leq$ age $<19$ & 68 & 1100 & 754.6 & $835 \cdot 3$ & 297.9 & $82 \cdot 8$ & 0.0 & $1205 \cdot 0$ & $376 \cdot 0$ & 43.7 & 0.0 & $1130 \cdot 8$ & 355.9 & $52 \cdot 0$ & 0.0 \\
\hline $19 \leq$ age $<31$ & 208 & 800 & $780 \cdot 0$ & $859 \cdot 4$ & $239 \cdot 3$ & $44 \cdot 3$ & 0.0 & $1239 \cdot 2$ & $343 \cdot 3$ & $7 \cdot 8$ & 0.3 & $1163 \cdot 4$ & 317.5 & $11 \cdot 0$ & 0.1 \\
\hline $31 \leq$ age $<51$ & 481 & 800 & $685 \cdot 7$ & $790 \cdot 2$ & 257.8 & $56 \cdot 8$ & 0.0 & 1132.9 & 363.5 & $17 \cdot 7$ & 0.2 & $1064 \cdot 2$ & 337.8 & $22 \cdot 5$ & 0.1 \\
\hline $51 \leq$ age $<71$ & 418 & 800 & $692 \cdot 3$ & 838.7 & 283.7 & $49 \cdot 6$ & 0.2 & $1182 \cdot 1$ & $366 \cdot 3$ & $13 \cdot 8$ & $2 \cdot 6$ & 1113.5 & 344.7 & $18 \cdot 0$ & 1.5 \\
\hline $71 \leq$ age & 105 & 1000 & $643 \cdot 8$ & $917 \cdot 2$ & $275 \cdot 0$ & $66 \cdot 2$ & 0.2 & $1243 \cdot 7$ & $376 \cdot 3$ & $27 \cdot 8$ & 3.9 & 1178.5 & 354.9 & $34 \cdot 0$ & 2.5 \\
\hline
\end{tabular}

EAR, estimated average requirement; UL, upper limit.

We calculated the usual Ca intake with the information from repeated measures except for Argentina, as the database had only one recall and for Lao and pregnant women of Italy, where the number of cases with duplicate recalls where small. In these cases, we used the 2003-2008 NHANES coefficient of variance provided by IMAPP as the external variance.

Below we report the results of the mean $\mathrm{Ca}$ intake, prevalence of low $\mathrm{Ca}$ intake and percentage of $\mathrm{Ca}$ intake above the upper limit for each of the IOM age groups before and after fortification.

\section{Uganda}

The mean daily Ca intake ranged from 363.3 (SD 163.2) to 462.7 (SD 283.7) $\mathrm{mg}$ in non-pregnant women and from $372 \cdot 3$ (SD 118.5) to $389 \cdot 3$ (SD 137.4) $\mathrm{mg}$ in pregnant women. The prevalence of low Ca intake was approximately $90 \%$ or higher in all population groups. None of the population age groups had $\mathrm{Ca}$ intake exceeding the recommended upper limits for $\mathrm{Ca}$ (Table 1).

After simulating the intake of water fortified with $500 \mathrm{mg}$ of $\mathrm{Ca} / 1$ assuming an intake of 1 litre of water/d, the prevalence of low Ca intake decreased to $31 \%$ in non-pregnant women aged 19 to $<31$ years, to $40.8 \%$ in non-pregnant women aged 31 to $<51$ years and to $70.8 \%$ in non-pregnant women aged 51 to $<71$ years (Table 1 ). After simulating the intake of water fortified with $500 \mathrm{mg}$ of $\mathrm{Ca} / \mathrm{l}$ assuming an intake of 1.5 litres of water/d, the prevalence of low Ca intake decreased to $0 \%$ in women aged 19 to $<<51$ years and to $22.1 \%$ in the group aged 51 to $<<71$ years. None of the groups had more than
$0.5 \%$ of the individuals with $\mathrm{Ca}$ intake exceeding the recommended upper limit for $\mathrm{Ca}$.

The original distribution of $\mathrm{Ca}$ intake and distribution of Ca intake after the simulation of water fortification with $500 \mathrm{mg}$ of $\mathrm{Ca} / \mathrm{l}$ assuming an intake of 1 litre of water/d are shown in online supplementary material, Supplemental Fig. S1.

\section{Lao People's Democratic Republic}

The mean daily Ca intake ranged from $214 \cdot 1$ (SD 104.6) to 364.1 (SD 257.8) $\mathrm{mg}$ in children, from 207.2 (SD 93.4) to 263.5 (SD 109.9) $\mathrm{mg}$ in girls and non-pregnant women, from 229.6 (SD 103.1) to 313.7 (SD 185.5) $\mathrm{mg}$ in pregnant women and from 212.0 (sD 62.5) to 435.6 (sD 318.3) $\mathrm{mg}$ in men. The prevalence of low Ca intake ranged from 58.4 to $99.5 \%$ in children and it was around $90 \%$ in the rest of the groups, and none of them had $\mathrm{Ca}$ intake higher than $2 \%$ of the upper limit (Table 2).

After simulating the intake of water fortified with $500 \mathrm{mg}$ of $\mathrm{Ca} / \mathrm{l}$ assuming an intake of 1 litre of water/d, the prevalence of low $\mathrm{Ca}$ intake decreased to $0 \%$ in children $<4$ years, to 24.8 in children aged 4 to $<9$ years, to between 45.0 and $90.8 \%$ in men aged 19 and over, to between 55.7 and $99.8 \%$ in pregnant women aged 14 to $<51$ years and to between 71.6 and $99.9 \%$ in women aged 14 to $<71$ years. (Table 2 ) None of the groups had more than $1.5 \%$ of the individuals with Ca intake exceeding the recommended upper limit for $\mathrm{Ca}$, except for infants $6-12$ months were the percentage exceeding the UL was $3 \cdot 2 \%$. 
Table 7 Simulation of the effect of water fortification with $500 \mathrm{mg}$ of calcium/l on calcium intake (USA)

\begin{tabular}{|c|c|c|c|c|c|c|c|c|c|c|c|c|c|c|c|c|c|c|c|c|c|c|c|}
\hline \multirow[b]{2}{*}{ Group } & \multirow[b]{2}{*}{$n$} & \multirow[b]{2}{*}{$\begin{array}{l}\text { EAR } \\
(\mathrm{mg})\end{array}$} & \multirow[b]{2}{*}{$\begin{array}{l}\text { Water } \\
\text { Intake }\end{array}$} & \multicolumn{4}{|c|}{ Basal Ca intake } & \multicolumn{4}{|c|}{$\begin{array}{l}\text { Ca intake after fortification of } \\
\text { water with } 500 \mathrm{mg} \text { of } \mathrm{Ca} / \mathrm{l}\end{array}$} & \multicolumn{4}{|c|}{$\begin{array}{l}\text { Ca intake after fortification of } \\
\text { water with } 400 \mathrm{mg} \text { of } \mathrm{Ca} / \mathrm{l}\end{array}$} & \multicolumn{4}{|c|}{$\begin{array}{l}\text { Ca intake after fortification of } \\
\text { water with } 300 \mathrm{mg} \text { of } \mathrm{Ca} / \mathrm{l}\end{array}$} & \multicolumn{4}{|c|}{$\begin{array}{l}\text { Ca intake after fortification of } \\
\text { water with } 200 \mathrm{mg} \text { of } \mathrm{Ca} / \mathrm{l}\end{array}$} \\
\hline & & & & $\begin{array}{c}\text { Mean } \\
(\mathrm{mg})\end{array}$ & SD & $\begin{array}{c}<\text { EAR } \\
(\%)\end{array}$ & $\begin{array}{l}>U L \\
(\%)\end{array}$ & $\begin{array}{c}\text { Mean } \\
(\mathrm{mg})\end{array}$ & SD & $\begin{array}{c}<\text { EAR } \\
(\%)\end{array}$ & $\begin{array}{l}>U L \\
(\%)\end{array}$ & $\begin{array}{c}\text { Mean } \\
(\mathrm{mg})\end{array}$ & SD & $\begin{array}{c}<\text { EAR } \\
(\%)\end{array}$ & $\begin{array}{l}>U L \\
(\%)\end{array}$ & $\begin{array}{c}\text { Mean } \\
(\mathrm{mg})\end{array}$ & SD & $\begin{array}{c}<\text { EAR } \\
(\%)\end{array}$ & $\begin{array}{l}>U L \\
(\%)\end{array}$ & $\begin{array}{c}\text { Mean } \\
(\mathrm{mg})\end{array}$ & SD & $\begin{array}{c}<\text { EAR } \\
(\%)\end{array}$ & $\begin{array}{c}>U L \\
(\%)\end{array}$ \\
\hline \multicolumn{24}{|l|}{ Children } \\
\hline $0 \leq$ age $<1$ & 222 & 270 & 44.5 & $650 \cdot 0$ & 288.4 & 3.7 & 1.7 & 671.7 & $299 \cdot 1$ & $3 \cdot 3$ & $2 \cdot 1$ & $667 \cdot 4$ & 296.4 & $3 \cdot 3$ & $2 \cdot 0$ & 663.0 & 294.0 & 3.4 & 1.9 & $658 \cdot 7$ & 291.9 & 3.5 & 1.9 \\
\hline $1 \leq$ age $<4$ & 564 & 400 & $289 \cdot 3$ & $915 \cdot 0$ & $492 \cdot 0$ & $11 \cdot 1$ & $1 \cdot 1$ & $1059 \cdot 0$ & $535 \cdot 3$ & 6.5 & $2 \cdot 0$ & $1030 \cdot 2$ & $522 \cdot 5$ & $7 \cdot 0$ & 1.7 & $1001 \cdot 4$ & 511.6 & $7 \cdot 7$ & 1.5 & $972 \cdot 6$ & $502 \cdot 8$ & 8.6 & $1 \cdot 3$ \\
\hline $4 \leq$ age $<9$ & 828 & 640 & 438.6 & $952 \cdot 3$ & $487 \cdot 6$ & 28.9 & 0.7 & 1171.4 & $556 \cdot 7$ & $16 \cdot 7$ & $2 \cdot 2$ & $1127 \cdot 6$ & 535.8 & $18 \cdot 2$ & 1.7 & $1083 \cdot 8$ & $518 \cdot 1$ & $20 \cdot 1$ & 1.3 & $1040 \cdot 0$ & 504.0 & 22.5 & 1.0 \\
\hline \multicolumn{24}{|c|}{ Women non-pregnant } \\
\hline $9 \leq$ age $<14$ & 417 & 1100 & 539.7 & $919 \cdot 1$ & 547.9 & $69 \cdot 2$ & 0.4 & $1188 \cdot 8$ & 643.9 & $51 \cdot 0$ & 1.3 & $1134 \cdot 8$ & $615 \cdot 2$ & $54 \cdot 3$ & 1.0 & $1080 \cdot 9$ & $590 \cdot 7$ & $58 \cdot 0$ & 0.7 & $1027 \cdot 0$ & 570.9 & $61 \cdot 8$ & 0.6 \\
\hline $14 \leq$ age $<19$ & 383 & 1100 & $775 \cdot 2$ & $826 \cdot 8$ & $520 \cdot 9$ & $75 \cdot 4$ & 0.3 & $1214 \cdot 2$ & 731.9 & $51 \cdot 7$ & $2 \cdot 6$ & $1136 \cdot 7$ & 674.1 & $55 \cdot 7$ & 1.7 & $1059 \cdot 2$ & $622 \cdot 2$ & $60 \cdot 3$ & 1.0 & 981.7 & 577.8 & $65 \cdot 3$ & 0.6 \\
\hline $19 \leq$ age $<31$ & 511 & 800 & $1242 \cdot 5$ & $825 \cdot 9$ & 501.5 & $56 \cdot 3$ & 0.9 & $1446 \cdot 8$ & 843.4 & 23.7 & $11 \cdot 2$ & $1322 \cdot 6$ & 750.7 & $26 \cdot 8$ & 7.5 & 1198.5 & $666 \cdot 2$ & $31 \cdot 0$ & 4.5 & 1074.3 & 593.0 & $37 \cdot 0$ & $2 \cdot 4$ \\
\hline $31 \leq$ age $<51$ & 854 & 800 & $1236 \cdot 1$ & 837.7 & 471.8 & 54.6 & 0.6 & $1455 \cdot 3$ & 786.9 & $21 \cdot 0$ & $10 \cdot 2$ & 1331.8 & 701.9 & $24 \cdot 1$ & $6 \cdot 6$ & $1208 \cdot 3$ & 624.0 & $28 \cdot 3$ & 3.7 & $1084 \cdot 8$ & 556.7 & 34.5 & 1.9 \\
\hline $51 \leq$ age $<71$ & 845 & 1000 & $1166 \cdot 5$ & $775 \cdot 7$ & 436.4 & 74.9 & 1.6 & 1358.5 & 736.0 & $35 \cdot 8$ & $17 \cdot \overline{6}$ & $1242 \cdot 0$ & 655.8 & 40.9 & $12 \cdot 6$ & 1125.4 & 582.2 & 47.4 & $8 \cdot 1$ & $1008 \cdot 8$ & 518.1 & $55 \cdot 7$ & 4.7 \\
\hline $71 \leq$ age & 381 & 1000 & 899.5 & 731.7 & $418 \cdot 3$ & $78 \cdot 6$ & 1.3 & $1180 \cdot 7$ & $627 \cdot 6$ & 44.2 & $10 \cdot 0$ & 1091.0 & $567 \cdot 1$ & 49.9 & $7 \cdot 0$ & $1001 \cdot 1$ & 512.4 & $56 \cdot 6$ & 4.5 & 911.3 & $467 \cdot 2$ & $64 \cdot 2$ & $2 \cdot 8$ \\
\hline \multicolumn{24}{|l|}{ Pregnant women } \\
\hline $19 \leq$ age $<31$ & 37 & 800 & 1574.4 & 999.0 & $542 \cdot 3$ & 40.5 & 1.2 & 1773.4 & 879.4 & 13.5 & $20 \cdot 1$ & 1618.4 & 777.4 & $15 \cdot 2$ & $13 \cdot 3$ & 1463.5 & 687.0 & $17 \cdot 6$ & $7 \cdot 3$ & $1308 \cdot 6$ & $612 \cdot 7$ & 21.6 & 3.3 \\
\hline $31 \leq$ age $<51$ & 26 & 800 & 1481.2 & 947.7 & 507.5 & $42 \cdot 8$ & 0.6 & $1690 \cdot 1$ & $1136 \cdot 1$ & 23.2 & $20 \cdot 7$ & 1541.4 & 978.3 & 24.4 & $15 \cdot 7$ & 1392.4 & 825.6 & $26 \cdot 2$ & $10 \cdot 1$ & 1244.2 & 684.5 & 28.4 & 4.8 \\
\hline \multicolumn{24}{|l|}{ Male } \\
\hline $9 \leq$ age $<14$ & 412 & 1100 & 579.2 & $1039 \cdot 3$ & 650.0 & $62 \cdot 2$ & 1.3 & $1328 \cdot 1$ & $760 \cdot 8$ & $45 \cdot 2$ & 3.5 & $1270 \cdot 4$ & $728 \cdot 2$ & $48 \cdot 1$ & $2 \cdot 8$ & $1212 \cdot 6$ & $700 \cdot 7$ & 51.5 & $2 \cdot 3$ & $1154 \cdot 8$ & $678 \cdot 1$ & $55 \cdot 1$ & 1.9 \\
\hline $14 \leq$ age $<19$ & 374 & 1100 & 859.2 & $1100 \cdot 3$ & 698.9 & $58 \cdot 1$ & 1.8 & $1529 \cdot 1$ & 963.5 & 38.9 & 7.9 & 1443.3 & 892.9 & 41.6 & $6 \cdot 1$ & $1357 \cdot 6$ & 828.7 & 44.9 & 4.6 & 1271.8 & 773.2 & $48 \cdot 7$ & 3.3 \\
\hline $19 \leq$ age $<31$ & 497 & 800 & $1367 \cdot 6$ & $1067 \cdot 7$ & $719 \cdot 6$ & $42 \cdot 9$ & 4.7 & $1751 \cdot 2$ & $1132 \cdot 4$ & 19.9 & 21.4 & 1614.6 & 895 & $1023 \cdot 6$ & $22 \cdot 0$ & 1477.9 & 924.0 & 24.9 & $13 \cdot 1$ & $1341 \cdot 3$ & 835.0 & $29 \cdot 0$ & 9.4 \\
\hline $31 \leq$ age $<51$ & 792 & 800 & 1318.9 & $1042 \cdot 1$ & $658 \cdot 1$ & $42 \cdot 7$ & 3.5 & 1701.4 & 988.3 & $17 \cdot 6$ & $18 \cdot 8$ & $1569 \cdot 6$ & $895 \cdot 2$ & $19 \cdot 7$ & $14 \cdot 4$ & $1437 \cdot 8$ & 811.8 & $22 \cdot 8$ & $10 \cdot 4$ & $1305 \cdot 9$ & 741.4 & $27 \cdot 2$ & $7 \cdot 2$ \\
\hline $51 \leq$ age $<71$ & 824 & 800 & 1054.9 & 939.1 & 581.7 & 47.9 & 5.8 & 1465.9 & $900 \cdot 1$ & 22.9 & $21 \cdot 7$ & 1360.6 & $814 \cdot 3$ & $25 \cdot 4$ & $17 \cdot 6$ & $1255 \cdot 2$ & $736 \cdot 4$ & $28 \cdot 8$ & $13 \cdot 7$ & $1149 \cdot 8$ & $669 \cdot 1$ & 33.5 & $10 \cdot 2$ \\
\hline $71 \leq$ age & 372 & 1000 & 779.4 & 871.6 & 553.8 & $67 \cdot 1$ & 3.9 & $1260 \cdot 6$ & $745 \cdot 7$ & $42 \cdot 3$ & 14.5 & 1182.5 & 689.1 & $46 \cdot 0$ & 11.5 & $1105 \cdot 1$ & $645 \cdot 2$ & 48.4 & $7 \cdot 8$ & $1027 \cdot 3$ & 604.0 & $53 \cdot 8$ & $5 \cdot 9$ \\
\hline
\end{tabular}

EAR, estimated average requirement; UL, upper limit. 
The original distribution of $\mathrm{Ca}$ intake and the distribution of $\mathrm{Ca}$ intake after the simulation of water fortification with $500 \mathrm{mg}$ of $\mathrm{Ca} / \mathrm{l}$ of water assuming an intake of 1 litre of water/d are shown in online supplementary material, Supplemental Fig. S2.

\section{Bangladesh}

The mean daily Ca intake ranged from 151.8 (sD 53.7 ) to 160.8 (SD 55.6) $\mathrm{mg}$ in non-pregnant women and from 143.2 (SD 80.3) to 150.2 (SD 58.8) $\mathrm{mg}$ in pregnant women. The prevalence of low $\mathrm{Ca}$ intake was $100 \%$ in all age groups. None of the groups had individuals with a $\mathrm{Ca}$ intake exceeding the recommended upper limit for $\mathrm{Ca}$ (Table 3).

After simulating the intake of water fortified with $500 \mathrm{mg}$ of $\mathrm{Ca} / \mathrm{l}$ and assuming an intake of 1 litre of water/d, the prevalence of low $\mathrm{Ca}$ intake decreased to around $95 \%$ in all age groups (Table 3). After simulation, the intake of water fortified with $500 \mathrm{mg}$ of $\mathrm{Ca} / \mathrm{l}$ and assuming an intake of 1.5 litres of water/d, the prevalence of low $\mathrm{Ca}$ intake decreased to nearly $0 \%$ in all age groups. None of the groups had more than $0.5 \%$ of the individuals with $\mathrm{Ca}$ intake exceeding the recommended upper limit for $\mathrm{Ca}$.

The original distribution of $\mathrm{Ca}$ intake and the distribution of $\mathrm{Ca}$ intake after the simulation of water fortification with $500 \mathrm{mg}$ of $\mathrm{Ca} / \mathrm{l}$ of water assuming an intake of 1 litre of water/d are shown in online supplementary material, Supplemental Fig. S3.

\section{Zambia}

The mean daily Ca intake ranged from 202.6 (SD $70 \cdot 8$ ) to 220.2 (SD 64.0) $\mathrm{mg}$ in children, from 311.5 (SD 24.5) to 339.3 (sD 9.6) $\mathrm{mg}$ in non-pregnant women and from 317.4 (sD 85.6) to 322.2 (sD 114.4) $\mathrm{mg}$ in pregnant women. The prevalence of low $\mathrm{Ca}$ intake was $98 \%$ or higher in all population groups. None of the groups had individuals with $\mathrm{Ca}$ intakes exceeding the recommended upper limit for $\mathrm{Ca}$ (Table 4).

After the simulation of water fortification with $500 \mathrm{mg} / \mathrm{l}$ assuming an intake of 1 litre of water/d, the prevalence of low $\mathrm{Ca}$ intake decreased to $<50 \%$ in all age groups (Table 4). After the simulation of water fortified with $500 \mathrm{mg}$ of $\mathrm{Ca} / \mathrm{l}$ and assuming an intake of 1.5 litres of water/d, the prevalence of low Ca intake decreased to $0 \%$ in all age groups. None of the groups had individuals with Ca intakes exceeding the upper limit.

The original distribution of $\mathrm{Ca}$ intake and the distribution of Ca intake after water fortification with $500 \mathrm{mg}$ of $\mathrm{Ca} / \mathrm{l}$ of water assuming an intake of 1 litre of water/d are shown in online supplementary material, Supplemental Fig. S4.

\section{Argentina}

The mean daily Ca intake ranged from 609.3 (SD 348.0) to 752.2 (SD 323.4) $\mathrm{mg}$ in children, from 361.6 (SD 180.2) to $460 \cdot 7$ (SD 167.6) $\mathrm{mg}$ in non-pregnant women and from
470.6 (SD 246.7) to 491.3 (SD 244.7) in pregnant women. The prevalence of low $\mathrm{Ca}$ intake was $17.5 \%$ in infants $<1$ year, $13.4 \%$ in children aged 1 to $<4$ years, $46.2 \%$ in children aged 4 to $<9$ years and $88 \%$ or higher in girls and women, including pregnant women. None of the groups had individuals with a $\mathrm{Ca}$ intake exceeding the recommended upper limit for $\mathrm{Ca}$, except for infants $<1$ year (Table 5).

After the simulation of water fortification with $500 \mathrm{mg} / \mathrm{l}$ assuming an intake of 1 litre of water/d, the prevalence of low $\mathrm{Ca}$ intake decreased to $0 \%$ in children $<4$ years, $0.4 \%$ in children aged 4 to $<9$ years. The prevalence of low $\mathrm{Ca}$ intake was reduced to around $80 \%$ in girls aged 9 to $<19$ years, to $30.4 \%$ in women aged 19 to $<31$ and to $42.7 \%$ in women aged 31 to $<51$ years. In pregnant women, the prevalence of low $\mathrm{Ca}$ intake was reduced to $75.6 \%$ in those aged 14 to $<19$ years, to $23.1 \%$ in those aged 19 to $<31$ years and to $24 \cdot 2 \%$ in those aged 31 to $<51$ years (Table 5). None of the groups had more than $0.5 \%$ of the individuals with $\mathrm{Ca}$ intakes exceeding the upper limit, except for infants aged 6 months to $<1$ year that had $6.1 \%$.

After the simulation of water fortified with $500 \mathrm{mg}$ of $\mathrm{Ca} / \mathrm{l}$ and assuming an intake of 1.5 litres of water/d, the prevalence of low $\mathrm{Ca}$ intake decreased to nearly $0 \%$ in most age groups except for girls aged 9 to $<14$ years $(27.6 \%$ ), for girls 14 to $<19$ years $(37.7 \%)$ and for pregnant girls aged $14-19$ years $(35.9 \%)$. None of the groups had more than $0.5 \%$ of the individuals with Ca intakes exceeding the upper limit, except for infants aged 6 months to $<1$ year that had $9.3 \%$.

The original distribution of Ca intake and the distribution of Ca intake after water fortification with $500 \mathrm{mg}$ of $\mathrm{Ca} / \mathrm{l}$ of water simulating the intake of 1 litre of water/d are shown in online Supplemental Fig. S5.

\section{Italy}

The mean daily $\mathrm{Ca}$ intake ranged from 577.0 (SD 127.5) to 728.6 (SD 180.5) $\mathrm{mg}$ in children, from 822.0 (SD 267.6) to 1009.1 (sD 181.2) $\mathrm{mg}$ in pregnant women, from 716.4 (237.5) to 819.5 (SD 187.0) $\mathrm{mg}$ in girls and non-pregnant women and from 790.2 (SD 257.8) to 917.2 (SD 275.0) $\mathrm{mg}$ in boys and men. The prevalence of low $\mathrm{Ca}$ intake ranged from 0.3 to $37.2 \%$ in children, from 13.1 to $51.0 \%$ in pregnant women, from 65.7 to 92.3 in girls and non-pregnant women and from 44.3 to $82.8 \%$ in boys and men. None of the groups had $>0.2 \%$ of the individuals with Ca intakes exceeding the recommended upper limit for $\mathrm{Ca}$ (Table 6).

After the simulation of water fortification with $500 \mathrm{mg} / \mathrm{l}$ taking into account the reported water intake of each individual, the prevalence of low Ca intake decreased to $<57 \%$ in all age groups; however, the percentage of individuals exceeding the upper limit increased to between 2.6 and $3.9 \%$ in men older than 51 years (Table 6). We then simulated a fortification with lower Ca concentrations. With 
Simulation of high calcium water on calcium intake

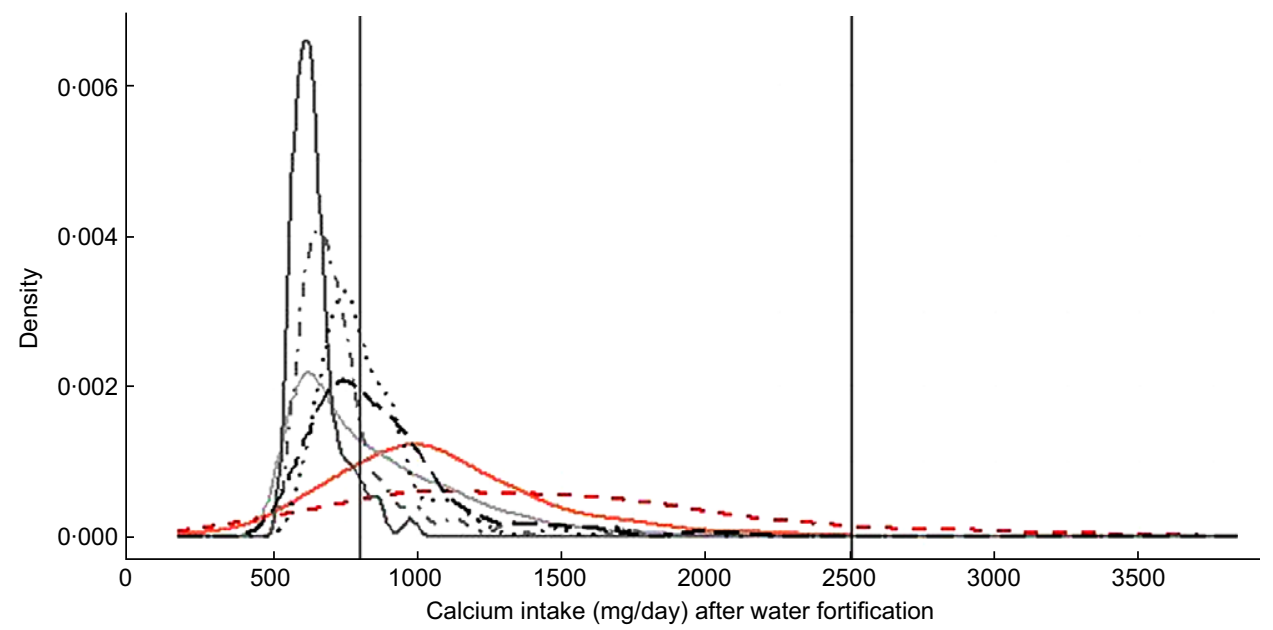

Fig. 1 Simulation of water fortification. Country: —, Argentina; -, Italy; —, Uganda; • •, Zambia; —, Bangladesh; ·-, Lao; --, USA

$400 \mathrm{mg}$ of $\mathrm{Ca} / \mathrm{l}$ of water, we found that the prevalence of inadequate $\mathrm{Ca}$ intake reached $64.7 \%$ in some age groups like boys and girls aged 9 to $<19$ years. On the other, the percentage of individuals exceeding the upper limit decreased to $1.5 \%$ in men aged 31 to $<51$ years and to $2.5 \%$ in men older than 51 years.

The original distribution of Ca intake and the distribution of $\mathrm{Ca}$ intake after water fortification are shown in online supplementary material, Supplemental Fig. S6.

\section{$\boldsymbol{U} \boldsymbol{S} \boldsymbol{A}$}

The mean daily Ca intake ranged from $650 \cdot 0$ (SD 288.4) to 952.3 (SD 487.6) $\mathrm{mg}$ in children, from 731.7 (SD 418.3) to $919 \cdot 1$ (SD 547.9) $\mathrm{mg}$ in girls and non-pregnant women, from 947.7 (SD 507.5) to 999.0 (SD 542.3) $\mathrm{mg}$ in pregnant women and from 871.6 (SD 553.8) to 1100.3 (SD 698.9) $\mathrm{mg}$ in boys and men. The prevalence of low Ca intake ranged from 3.7 to $28.9 \%$ in children, from 54.6 to $78.6 \%$ in girls and nonpregnant women, from 40.5 to $42.8 \%$ in pregnant women and from 42.9 to $67.1 \%$ in boys and men. Children and women did not exceed the $\mathrm{Ca}$ intake upper limit in $>2 \%$, whereas around $3 \cdot 5-5.8 \%$ of the individuals exceeded the upper limit in the group of men older than 31 years (Table 7 ).

After the simulation of water fortification with $500 \mathrm{mg} / \mathrm{l}$ and taking into account the reported water intake of each individual, the prevalence of low Ca intake decreased in all age groups; however, the percentage of individuals exceeding the upper limit increased to $20 \%$ in pregnant women and between 14.5 and $21.7 \%$ in males older than 19 years (Table 7 ). We then simulated a fortification with 400 and $300 \mathrm{mg}$ of $\mathrm{Ca} / \mathrm{l}$ of water and found that pregnant women and young men groups still had around $10 \%$ of individuals exceeding the upper limit. Finally, we simulated the fortification with $200 \mathrm{mg}$ of $\mathrm{Ca} / \mathrm{l}$ of water and found that the prevalence of inadequate intake was still high in men aged 51 to $<71$ years; however, the percentage of individuals exceeding the upper limit decreased to $10 \%$ or less.

The original distribution of Ca intake and the distribution of Ca intake after simulating water fortification are shown in online Supplemental Fig. S7.

Finally, Fig. 1 presents the distribution of Ca intake of pregnant women aged between 31 and 50.9 years after simulating the fortification of water with $500 \mathrm{mg}$ of $\mathrm{Ca} / \mathrm{l}$. For Argentina, Lao, Uganda, Zambia and Bangladesh, the simulation assumes that each woman drinks 1 litre of water/d, whereas for Italy and USA, the simulation takes into account the individual water intake. The figure shows that after water fortification, most of the population of HIC had Ca intake between 800 and $2500 \mathrm{mg}$ of $\mathrm{Ca}$ a day which are the limits considered safe for $\mathrm{Ca}$, whereas all LMIC had most of their populations under $800 \mathrm{mg}$ of $\mathrm{Ca} / \mathrm{d}$.

\section{Discussion}

The current study analyses the adequacy of $\mathrm{Ca}$ intake in populations from Uganda, Zambia, Bangladesh, Lao PDR, Argentina, Italy and USA. It describes the usual Ca intake, the prevalence of low $\mathrm{Ca}$ intake and the percentage of individuals with $\mathrm{Ca}$ intake above the upper limit using the IMAPP developed by IOWA. We then described the changes in the $\mathrm{Ca}$ intake distribution for each IOM age group after the simulation of a water fortification strategy.

In the current study, we found that for all the dietary databases assessed from LMIC, the strategy of fortifying water with $500 \mathrm{mg}$ of $\mathrm{Ca} / \mathrm{l}$ would decrease the prevalence of low $\mathrm{Ca}$ intake in all age groups. We also found that this strategy in the LMIC assessed would be safe as no group would present a percentage of individuals exceeding the upper limit in more than $2 \%$. In Italy, a strategy of fortifying water with $500 \mathrm{mg}$ of $\mathrm{Ca} / \mathrm{l}$ would lead to between 2.6 and $3.9 \%$ of men aged 31 years or more exceeding the upper limit. We simulated fortifying water with $400 \mathrm{mg}$ of $\mathrm{Ca} / \mathrm{l}$ 
and found that although it would decrease the prevalence of low Ca intake, it would leave $2.5 \%$ of men aged 51 years or older exceeding the upper limit for $\mathrm{Ca}$ intake.

On the other hand, in the USA, even though there were groups with high prevalence of low Ca intake, a strategy to fortify water with Ca would lead to safety issues. In the USA, water fortified with $200 \mathrm{mg}$ of $\mathrm{Ca} / \mathrm{l}$ would lead to a decrease in the prevalence of low Ca intake; however, some groups would have exceeded the upper limit in around $10 \%$. Our analysis shows that in countries like the USA, even when the water intake is lower than recommended, a populationbased policy to fortify water would not be recommended. Strategies to increase Ca should focus on specific population groups with low Ca intake.

The current study uses information from different countries with diverse age groups including pregnant women, which gives strength to the results. Most data were collected using duplicate $24 \mathrm{~h}$ recalls or food records that are validated methodologies to measure $\mathrm{Ca}$ intake ${ }^{(33)}$. Only one country, Argentina, had a single $24 \mathrm{~h}$ recall that as recommended was adjusted with external variability to obtain estimates of Ca inadequacy, the complex sampling of this database was also taken into account in the analysis $^{(34)}$.

One limitation of the current study is that dietary surveys from Uganda, Lao PDR, Bangladesh, Zambia and Argentina did not collect water intake for each individual in the sample. In this way, if actual water intake was less than the simulated 1 litre/d, the reduction of Ca intake inadequacy would be lower than the one shown here. Furthermore, as water was not registered in the surveys, the basal Ca provided by local water intake was not calculated. We did not find data on the Ca concentration of water from Uganda, Lao PDR, Bangladesh or Zambia to estimate if this could impact the total Ca intake estimated with these databases. In Argentina, there is evidence from pregnant women that water could contribute to around $6 \%$ of total $\mathrm{Ca}$ intake; however, due to the large prevalence of low $\mathrm{Ca}$ intake, this information would not change the results presented in the current study ${ }^{(35)}$

Another limitation of the current study is that we were not able to assess the across person variability of water intake for most of the countries as water consumption was simulated for each individual in these databases; thus, the real distribution of Ca intake would probably be different from the one presented here. However, although we did not know the exact amount of water intake, water is generally consumed by all individuals and on a daily basis ${ }^{(36)}$. A further limitation of the current study is the small sample size in some age groups so for those specific groups the results should be interpreted with caution.

We calculated the percentage of individuals above the upper limit of $\mathrm{Ca}$ intake for infants $<1$ year taking into account recommendations of water intake as it is unlikely that they consume 1 litre of water a day, especially those under 6 months were the recommendation is exclusive breastfeeding ${ }^{(37)}$. Recommendations of water intake from beverages, excluding water from foods, are 600 to $800 \mathrm{ml} / \mathrm{d}$ for infants aged 6 to $<12$ months, $900 \mathrm{ml}$ to 1 litre for children aged 1 to $<4$ years, 1.2 litres for children aged 4 to $<9$ years, 1.5-1.8 litres/d for older children and $1.5-2$ litres/d for adults and pregnant women ${ }^{(38,39)}$. Taking into account the USA data analysed in the current study, daily water intake seems to be lower than the recommended amounts ${ }^{(29)}$. Studies from Britain, Spain and Italy also show that water intake is lower than the recommended ${ }^{(40,41)}$.

Water intake information is not always collected in dietary surveys as shown by the analysis of these databases. For this article, we searched national or subnational databases with information of daily food and nutrient obtained from $24 \mathrm{~h}$ recalls or daily food records. Of the databases analysed, only two had data on water intake. These two databases were from HIC and none from LMIC. The lack of data on water intake and Ca concentration of water in the populations of LMIC included in the current study are the main limitations of this study. Also, there is limited information on the type of drinking water as many dietary surveys do not collect these data in detail ${ }^{(40)}$. Water intake is difficult to estimate, and there is no standardised form to collect these data. Information on the amount and source of water consumed would be required to better tailor a water fortification strategy for a particular population.

Although, the current study does not take in account special groups with extreme water intake that could be put at risk of excess Ca intake with a water fortification strategy, we found that even in HIC, very few people consumed more than 1.5 litres of water a day. In Italy, only $4.4 \%$ $(56 / 1280)$ of men and $4.3 \%(69 / 1612)$ of women aged 14 years or more had intakes higher than 1.5 litres of water. In USA, $26 \cdot 8 \%(767 / 2859)$ of men and $27.4 \%(832 / 3037)$ of women aged 14 years or higher had intakes higher than 1.5 litres of water.

Studies have refuted some Ca supplementation side effects like detriment of iron status, formation of renal stones and myocardial infarction in older people. However, in some supplementation studies, a few deleterious effects, such as postpartum bone resorption and gastrointestinal discomfort, have been reported.

One study showed that the consumption of Ca-rich water can provide a quarter of total Ca daily intake and it can be a recommendation to increase $\mathrm{Ca}$ intake in people with low intake of dairy products ${ }^{(42)}$. Another study from Poland showed that tap water with around $68-114 \mathrm{mg}$ of $\mathrm{Ca} / \mathrm{l}$ can contribute to $6-14 \%$ of total $\mathrm{Ca}$ intake of young women. Finally, a study from the UK showed that Ca intake in areas where Ca concentration of water reaches $300 \mathrm{mg}$ of $\mathrm{Ca} / 1$ can contribute to $8 \%$ of adolescents total Ca intake $^{(43,44)}$.

Mineral waters with high concentration of $\mathrm{Ca}$, around $300 \mathrm{mg} / \mathrm{l}$ or more, are found in Italy, Spain, the UK and France ${ }^{(44-46)}$. Ca concentration of artificially mineral bottled waters is much lower, and technical research to increase Ca 
content of artificially mineral and or tap water is required to improve $\mathrm{Ca}$ intake of low $\mathrm{Ca}$ intake populations from areas where local water has low Ca concentrations ${ }^{(47)}$.

WHO recommends a minimum of $20 \mathrm{mg}$ of $\mathrm{Ca} / \mathrm{l}$ of water and to prevent risk of CVD suggest an optimal concentration between 50 and $80 \mathrm{mg} / \mathrm{l}^{(48)}$. In this way, Ca concentration of water should be regulated more tightly as it could be a good source of minerals for the diet. Regulations should control a minimum level of Ca in tap water so as to improve health, specially taking into account that waters with low $\mathrm{Ca}$ and magnesium could be corrosive and harmful ${ }^{(49)}$

Currently, tap water regulations include a maximum level of $\mathrm{Ca}$ concentration, while fewer include a minimum Ca concentration, which if so they usually focus on hardness. Furthermore, $\mathrm{Ca}$ concentration values are usually provided for technical rather than health considerations ${ }^{(48)}$. In some US states, the minimum Ca concentration required is $10 \mathrm{mg} / \mathrm{l}$; however, based on health concerns, WHO recommends Ca concentrations of no $<20-30 \mathrm{mg} / \mathrm{l}$ with an optimal amount of $40-80 \mathrm{mg} / \mathrm{l}^{(49-51)}$. We have reviewed international and national food and beverage regulations, and the policy to add Ca to water is present only in those areas with demineralised and/or desalinated water ${ }^{(48)}$. Our results show that $\mathrm{Ca}$ in water can improve the diet of populations with low $\mathrm{Ca}$ intake and thus we consider that setting a minimum $\mathrm{Ca}$ concentration level in water could contribute to attain an adequate $\mathrm{Ca}$ intake and its health benefits $^{(52)}$ help to improve the health of those populations with low Ca intake.

After these preliminary results, if water fortification is feasible, the implementation of this policy would require in each particular population the assessments of infrastructure, water sources, funds, local regulations and acceptability (such as change in taste) according to the selected fortification process.

\section{Conclusion}

We found that for most LMIC countries, increasing Ca concentration of water to $500 \mathrm{mg}$ of $\mathrm{Ca} / \mathrm{l}$ would decrease the prevalence of $\mathrm{Ca}$ intake inadequacy without exceeding the upper level of $\mathrm{Ca}$ intake in any population group. On the other hand, if the water intake is 1.5 litres a day, the amount of Ca would still be safe in LMIC, except for women aged 19-31 years in Lao PDR.

The current study confirms that low $\mathrm{Ca}$ intake can also be found within some population age groups of HIC. In USA, Ca intake has a widespread distribution, implying that some individuals have very low or very high intakes within the same age group. In similar situations where Ca intake levels are extreme such as in the USA, a strategy reaching those specific groups should be put in place.

Food fortification is the most cost-effective intervention to improve micronutrient intake; however, a comprehensive evaluation of its impact should include a simulation analysis like the one presented.

The current study reinforces the need to plan Ca fortification strategies given the health relevance of an adequate $\mathrm{Ca}$ intake and the high percentage of individuals in LMIC who fail to meet the requirements. Water is an interesting vehicle because it is of universal and equitable intake showing a good bioavailability that does not imply an increase in energy intake that can contribute to obesity. Furthermore, unlike other foods, no population group has dietary restrictions to water.

Fortifying water can only work if the population uses water that can be treated, for example, tap or bottled water and not collected from natural sources. Research to achieve fortification of different drinking water sources with $\mathrm{Ca}$ is fully warranted. Strategies to add Ca to water networks, water wells, bottled water and possibility home devices to increase Ca concentration of water deserve to be investigated and tested to reach the entire population.

\section{Acknowledgements}

Acknowledgements: The authors would like to thank Alicia Carriquiry for her support and training on the use of IMAPP and Enrique Abeyá, Pablo Duran and Catherine Leclercq for their help in obtaining some of the databases. Financial support: The current research was funded by Bill \& Melinda Gates Foundation grant no. OPP1190821. Conflict of interest: There are no conflicts of interest. Authorship: All authors participate in the design, analysis, interpretation of the results and writing of the article. Ethics of buman subject participation: The current study is a secondary analysis of publicly available databases. Databases did not contain any personal information.

\section{Supplementary material}

For supplementary materials accompanying this paper visit https://doi.org/10.1017/\$1368980020002232.

\section{References}

1. Hofmeyr GJ \& Manyame S (2014) Calcium supplementation commencing before or early in pregnancy, or food fortification with calcium, for preventing hypertensive disorders of pregnancy. Cochrane Database Syst Rev. Published online: 2014. doi: 10.1002/14651858.CD011192.

2. Cormick G, Ciapponi A, Cafferata ML et al. (2015) Calcium supplementation for prevention of primary hypertension. Cochrane Database Syst Rev. Published online: 2015. doi: 10.1002/14651858.CD010037.pub2.

3. Bergel E \& Barros AJD (2007) Effect of maternal calcium intake during pregnancy on children's blood pressure: a systematic review of the literature. BMC Pediatr. Published online: 2007. doi: 10.1186/1471-2431-7-15. 
4. Bergel E \& Belizán JM (2002) A deficient maternal calcium intake during pregnancy increases blood pressure of the offspring in adult rats. BJOG An Int J Obstet Gynaecol. Published online: 2002. doi: 10.1111/j.1471-0528.2002.01155.x.

5. Hofmeyr GJ, Betrán AP, Singata-Madliki M et al. (2019) Prepregnancy and early pregnancy calcium supplementation among women at high risk of pre-eclampsia: a multicentre, double-blind, randomised, placebo-controlled trial. Lancet 393, 330-339.

6. Villa-Etchegoyen C, Lombarte M, Matamoros N et al. (2019) Mechanisms involved in the relationship between low calcium intake and high blood pressure. Nutrients 11, 1112.

7. Chen C, Ge S, Li S et al. (2017) The effects of dietary calcium supplements alone or with vitamin D on cholesterol metabolism. J Cardiovasc Nurs 32, 496-506.

8. Weingarten MA, Zalmanovici A \& Yaphe J (2008) Dietary calcium supplementation for preventing colorectal cancer and adenomatous polyps. Cochrane Database Syst Rev. Published online: 2008. doi: 10.1002/14651858.CD003548. pub4.

9. Zhang N, Wilkinson S, Riaz M et al. (2012) Calcium supplementation and kidney stone risk in osteoporosis? A systematic literature review. Clin Exp Rheumatol 30, 962-971.

10. Imdad A \& Bhutta ZA (2012) Effects of calcium supplementation during pregnancy on maternal, fetal and birth outcomes. Paediatr Perinat Epidemiol 26, 138-152.

11. Balk EM, Adam GP, Langberg VN et al. (2017) Global dietary calcium intake among adults: a systematic review. Osteoporos Int 28, 3315-3324.

12. Cormick G, Betrán AP, Romero IB et al. (2018) Global inequities in dietary calcium intake during pregnancy: a systematic review and meta-analysis. BJOG An Int J Obstet Gynaecol. Published online: 2018. doi: 10.1111/1471-0528. 15512 .

13. Institute of Medicine (1997) Dietary Reference Intakes For Calcium, Phosphorus, Magnesium, Vitamin D, and Fluoride. Washington, DC: The National Academies Press. doi: $10.17226 / 5776$.

14. Charlton K, Ware LJ, Baumgartner J et al. (2018) How will South Africa's mandatory salt reduction policy affect its salt iodisation programme? A cross-sectional analysis from the WHO-SAGE Wave 2 Salt and Tobacco study. BMJ Open. Published online: 2018. doi: 10.1136/bmjopen-2017-020404.

15. Lockyer S \& Spiro A (2020) The role of bread in the UK diet: an update. Nutr Bull 45, 133-164.

16. Hofmeyr GJ, Lawrie TA, Atallah ÁN et al. (2014) Calcium supplementation during pregnancy for preventing hypertensive disorders and related problems. Cochrane Database Syst Rev. Published online: 2014. doi: 10.1002/14651858. CD001059.pub4.

17. Bailey RL, Fulgoni VL, Keast DR et al. (2011) Dietary supplement use is associated with higher intakes of minerals from food sources. Am J Clin Nutr 94, 1376-1381.

18. Allen L, Benoist B de, Dary O et al. (2006) Guidelines on Food Fortification with Micronutrients. Geneva: WHO.

19. Iheozor-Ejiofor Z, Worthington HV, Walsh T et al. (2015) Water fluoridation for the prevention of dental caries. Cochrane Database Syst Rev. Published online: 18 June 2015. doi: 10.1002/14651858.CD010856.pub2.

20. Böhmer H, Mü ller H \& Resch KL (2000) Calcium supplementation with calcium-rich mineral waters: a systematic review and meta-analysis of its bioavailability. Osteoporos Int 11, 938-943.

21. Heaney RP \& Dowell MS (1994) Absorbability of the calcium in a high-calcium mineral water. Osteoporos Int $\mathbf{4}$, 323-324.

22. Wynckel A, Hanrotel C, Wuillai A et al. (1997) Intestinal calcium absorption from mineral water. Miner Electrolyte Metab 23, 88-92.
23. Wiseman M (1992) The COMA report: dietary reference values for food energy and nutrients for the United Kingdom. Br Food J 94, 7-9.

24. Guillemant J, Le HT, Accarie C et al. (2000) Mineral water as a source of dietary calcium: acute effects on parathyroid function and bone resorption in young men. Am J Clin Nutr 71, 999-1002.

25. World Health Organization (2010) Estimating Appropriate Levels of Vitamins and Minerals for Food Fortification Programmes: The WHO Intake Monitoring, Assessment and Planning Program (IMAPP): Meeting Report. Geneva: WHO; available at http://www.who.int/nutrition/publications/ micronutrients/9789241599603.pdf (accessed July 2020).

26. Morimoto JM, Marchioni DML, Cesar CLG et al. (2011) Within-person variance for adjusting nutrient distribution in epidemiological studies. Rev Saude Publica 45, 621-625.

27. Leclercq C, Allemand P, Balcerzak A et al. (2019) FAO/WHO GIFT (Global Individual Food consumption data Tool): a global repository for harmonised individual quantitative food consumption studies. Proc Nutr Soc. Published online: 2019. doi: 10.1017/S0029665119000491.

28. Ministry of Health from Argentina (2007) National Survey of Nutrition and Health. Encuesta Nacional de Nutrición y Salud (ENNyS). http://www.extensioncbc.com.ar/wp-content/ uploads/ENNyS-2007.pdf (accessed July 2020).

29. Centers for Disease Control and Prevention (CDC). National Health and Nutrition Examination Survey (NHANES) 20152016 Dietary Data. https://wwwn.cdc.gov/nchs/nhanes/ search/datapage.aspx?Component=Dietary\&CycleBegin Year $=2015$ (accessed July 2020).

30. Stray-Pedersen M, Helsing RM, Gibbons L et al. (2009) Weight status and hypertension among adolescent girls in Argentina and Norway: data from the ENNyS and HUNT studies. BMC Public Health. Published online: 2009. doi: 10.1186/1471-2458-9-398.

31. Sette S, Le Donne C, Piccinelli R et al. (2011) The third Italian National Food Consumption Survey, INRAN-SCAI 2005-06 Part 1: nutrient intakes in Italy. Nutr Metab Cardiovasc Dis 21, 922-932.

32. Institute of Medicine Committee to Review Dietary Reference Intakes for Calcium and Vitamin D (2011) Dietary Reference Intakes for Calcium and Vitamin D [AC Ross, CL Taylor, AL Yaktine et al., editors]. Washington, DC, USA: National Academies Press (US) National Academy of Sciences.

33. De Keyzer W, Huybrechts I, De Vriendt V et al. Repeated 24hour recalls versus dietary records for estimating nutrient intakes in a national food consumption survey. Food Nutr Res 55, 7307. doi: 10.3402/fnr.v55i0.7307.

34. Dodd KW, Guenther PM, Freedman LS et al. (2006) Statistical methods for estimating usual intake of nutrients and foods: a review of the theory. JAm Diet Assoc. Published online: 2006. doi: S0002-8223(06)01705-6.

35. Cormick G, Zhang NN, Andrade SP et al. (2014) Gaps between calcium recommendations to prevent preeclampsia and current intakes in one hospital in Argentina. BMC Res Notes 7, 920.

36. Rosinger AY, Herrick KA, Wutich AY et al. (2018) Disparities in plain, tap and bottled water consumption among US adults: National Health and Nutrition Examination Survey (NHANES) 2007-2014. Public Health Nutr 21, 1455-1464.

37. World Health Organization (2002) The optimal duration of exclusive breastfeeding. Trop Doct 32, 62-63.

38. EFSA Panel on Dietetic Products, Nutrition and A (NDA)2 (2010) Scientific opinion on dietary reference values for water. EFSA J. Published online: 2010. doi: 10.2903/j.efsa. 2010.1459 .

39. Gandy J (2015) Water intake: validity of population assessment and recommendations. Eur J Nutr 54, 11-16. 
40. Gibson S, Gunn P \& Maughan RJ (2012) Hydration, water intake and beverage consumption habits among adults. Nutr Bull 37, 182-192.

41. Nissensohn M, Sánchez-Villegas A, Ortega RM et al. (2016) Beverage consumption habits and association with total water and energy intakes in the Spanish population: findings of the ANIBES study. Nutrients $\mathbf{8}, 232$.

42. Galan P, Arnaud MJ, Czernichow S et al. (2002) Contribution of mineral waters to dietary calcium and magnesium intake in a French adult population. J Am Diet Assoc 102, 1658-1662.

43. Januszko O, Madej D, Postaleniec E et al. (2012). Minerals intake from drinking water by young women. Rocz Panstw Zakl Hig 63, 43-50.

44. Moynihan P, Adamson A, Rugg A et al. (1996) Dietary sources of calcium and the contribution of flour fortification to total calcium intake in the diets of Northumbrian adolescents. Br J Nutr 75, 495-505.

45. Galan P, Arnaud MJ, Czernichow S et al. (2002) Contribution of mineral waters to dietary calcium and magnesium intake in a French adult population. J Am Diet Assoc 102, $1658-1662$.

46. Vitoria I, Maraver F, Ferreira-Pêgo C et al. (2014) The calcium concentration of public drinking waters and bottled mineral waters in Spain and its contribution to satisfying nutritional needs. Nutr Hosp. Published online: 2014. doi: 10.3305/nh. 2014.30.1.7491.

47. Azoulay A, Garzon P \& Eisenberg MJ (2001) Comparison of the mineral content of tap water and bottled waters. J Gen Intern Med. Published online: 2001. doi: 10.1111/j.15251497.2001.04189.x.

48. World Health Organization (2017) Guidelines for DrinkingWater Quality, 4th ed., incorporating the first addendum. Geneva: World Health Organization. Licence: CC BY-NCSA 3.0 IGO.

49. World Health Organization. Sustainable Development and Healthy Environments Cluster (2005) Nutrients in Drinking Water. Geneva: World Health Organization. https://apps.who.int/iris/handle/10665/43403 (accessed July 2020)

50. United States Environmental Protection Agency (1998) The Quality of Our Nation's Water. Washington: Office of Water.

51. Koži冈? šek F Health Significance of Drinking Water Calcium and Magnesium (2003). http://www.szu.cz/uploads/ documents/chzp/voda/pdf/hardness.pdf (accessed July 2020).

52. Cormick G \& Belizán JM (2019) Calcium intake and health. Nutrients 11, 1606. Published online: 2019. doi: 10.3390/ nu11071606. 